\title{
Estimating the proportion of asymptomatic COVID- 19 cases in an Italian region with intermediate incidence during the first pandemic wave: an observational retrospective study
}

\section{Domenico Martinelli}

Department of Medical and Surgical Sciences, University of Foggia; Department of Hygiene, Policlinico Riuniti di Foggia, Foggia, Italy https://orcid.org/0000-0001-8028-3167

\section{Francesca Fortunato}

Department of Medical and Surgical Sciences, University of Foggia; Department of Hygiene, Policlinico Riuniti di Foggia, Foggia, ItalyDepartment of Medical and Surgical Sciences, University of Foggia; Department of Hygiene, Policlinico Riuniti di Foggia, Foggia, Italy https://orcid.org/0000-0003-06655385

\section{Sara Mazzilli}

Department of Translational Research and New Technologies in Medicine and Surgery, University of Pisa, Pisa, Italy; Scuola Normale Superiore, Pisa, Italy

\section{Lucia Bisceglia}

Strategic Regional Health and Social Agency of Puglia (AReS Puglia), Bari, Italy

\section{Pier Luigi Lopalco}

Department of Translational Research and New Technologies in Medicine and Surgery, University of Pisa, Pisa, Italy https://orcid.org/0000-0002-3181-8054

Rosa Prato ( $\sim$ rosa.prato@unifg.it)

Department of Medical and Surgical Sciences, University of Foggia; Department of Hygiene, Policlinico Riuniti di Foggia, Foggia, Italy https://orcid.org/0000-0001-8138-5835

\section{Research Article}

Keywords: Asymptomatic, COVID-19, SARS-CoV-2, Infection, Italy

Posted Date: December 17th, 2021

DOl: https://doi.org/10.21203/rs.3.rs-93013/v2

License: (c) (1) This work is licensed under a Creative Commons Attribution 4.0 International License. Read Full License 
Version of Record: A version of this preprint was published at BioMed Research International on January 6th, 2022. See the published version at https://doi.org/10.1155/2022/3401566. 


\section{Estimating the proportion of asymptomatic COVID-19 cases in an Italian region with intermediate incidence during the first pandemic wave: an observational retrospective study}

Domenico Martinelli ${ }^{1}$, Francesca Fortunato ${ }^{1}$, Sara Mazzilli ${ }^{2,3}$, Lucia Bisceglia ${ }^{4}$, Pier Luigi Lopalco $^{5}$, Rosa Prato ${ }^{1 *}$

${ }^{1}$ Policlinico Riuniti Foggia Hospital, Hygiene Unit, Department of Medical and Surgical Sciences, University of Foggia, Foggia, Italy

2 Department of Translational Research and New Technologies in Medicine and Surgery, University of Pisa, Pisa, Italy

${ }^{3}$ Scuola Normale Superiore, Pisa, Italy

${ }^{4}$ Strategic Regional Health and Social Agency of Puglia (AReSS Puglia), Bari, Italy

${ }^{5}$ Department of Biological and Environmental Sciences and Technology, University of Salento, Lecce, Italy

Correspondence should be addressed to: Prato Rosa, rosa.prato@unifg.it, Ospedale “Colonnello D’Avanzo", Viale degli Aviatori, 2 - 71122 Foggia, Italy, Tel.: +39-0881733091 


\begin{abstract}
Early in the COVID-19 pandemic, asymptomatic transmission represented an important challenge for controlling the spread of SARS-CoV-2 through the traditional public health strategies. Further understanding of the contribution of asymptomatic infections to SARS$\mathrm{CoV}-2$ transmission has been of crucial importance for pandemic control. We conducted a retrospective epidemiological study to characterize asymptomatic COVID-19 cases occurred in the Apulia region, Italy, during the first epidemic wave of COVID-19 outbreak (February 29 - July 7, 2020). We analyzed data collected in a regional platform developed to manage surveillance activities, namely investigation and follow-up of cases and contacts, contact tracing, laboratory and clinical data collection. We included all asymptomatic cases that were laboratory-confirmed during the appropriate follow-up, defined as persons infected with SARS-CoV-2 who did not develop symptoms/clinical signs of the disease. Between February 29 and July 7, 2020, a total of 4,536 cases were diagnosed with COVID-19 among 193,757 tests performed. The group of persons with asymptomatic SARS-CoV-2 infection consisted of 903 cases; the asymptomatic proportion was 19.9\% (95\%CI: 18.8-21.1\%); this decreased with increasing age (OR: $0.89,95 \% \mathrm{CI}$ : $0.83-0.96 ; \mathrm{p}=0.001$ ), in individuals with underlying comorbidities (OR: 0.55, 95\%CI: 0.41-0.73; $\mathrm{p}<0.001$ ), and in males (OR: 0.69, 95\%CI: 0.54 $0.87 ; \mathrm{p}=0.002$ ). The median asymptomatic SARS-CoV-2 RNA positive period was 19 days (IQR: 14-31) and the cumulative proportion of persons with resolution of infection 14 days after the first positive PCR test was $74 \%$. As the public health community is debating the question of whether asymptomatic and late spreaders could sustain virus transmission in the communities, such cases present unique opportunities to gain insight into SARS-CoV-2 adaptation to human host. This has important implications for future COVID-19 surveillance and prevention.
\end{abstract}




\section{Introduction}

SARS-CoV-2 is a novel coronavirus causing the current pandemic [1], which has resulted in millions of infections and hundreds of thousands of deaths worldwide. A total of 11,500,302 coronavirus disease 2019 (COVID-19) cases were diagnosed in the world during the initial wave of the epidemic (as of July 7, 2020). Exactly 137 days since the first confirmed COVID-19 case was announced, Italy was the eleventh most affected country, with 241,819 total cases and 34,869 deaths [2], mainly concentrated in the Northern area of the country, particularly in Lombardy, Piedmont, Emilia-Romagna, Veneto, and Liguria (80\% of cases diagnosed at national level). By contrast, the Covid-19 cumulative incidence remained substantially lower in the central and southern regions $[3,4]$.

The clinical outcomes of SARS-CoV-2 vary from asymptomatic infection to a mild to severe or critical disease [5-6].

Since COVID-19 burst onto the global scene, asymptomatic transmission of SARS-CoV-2 has appeared as the "Achilles' heel" of Covid-19 pandemic control through the traditional public health interventions [7]. Worldwide, the proportion of asymptomatic infection could be estimated to be 10.1-23.0\% of all confirmed cases before May 2020 [8]. It was reported that asymptomatic infections were more common in middle-aged individuals in Shenzhen (median age: 49 years; $30.9 \%$ between 30 and 49 years) and a few younger people in Nanjing (median age: 32.5 years) [9].

Further understanding of the transmission potential of asymptomatic individuals has been crucial to improve surveillance and containment measures and estimate the likely burden of severe disease and mortality when the virus spreads in the communities [10]. However, information on the natural history of infection with SARS-CoV-2 has yet to be fully described for quantifying the contribution of persons with asymptomatic COVID-19 infection to COVID-19 transmission [11].

Here, we conducted a retrospective epidemiological study to quantify and characterize asymptomatic COVID-19 cases occurred in the Apulia region of the southern part of Italy from February 29 to July 7, 2020.

\section{Materials and Methods}

We analyzed data collected in a regional surveillance platform (GIAVA-COVID ${ }^{\odot}$ ) developed on the basis of the Go.Data outbreak investigation tool (WHO) [12] to manage the emergency. GIAVA-COVID ${ }^{\odot}$ included functionalities for investigation and follow-up of cases (until having two consecutive negative RT-PCR test results at least 24 hours apart) and contacts (during the 14-day isolation period), contact tracing, demographics, laboratory and clinical data collection. The collected information included age, sex, residence location, date of disease onset, date of diagnosis, date of hospital admission, date of COVID-19, test results (positive or negative), date of death, presence of underlying diseases, case outcomes (hospitalisation, virus clearance and death) and disease severity (mild, moderate, severe or critical) [6]. The disease classification was duly updated according to clinical evolution of each case. 
This study included all asymptomatic cases, laboratory-confirmed by RT-PCR on nasopharyngeal swabs [13] that were sampled and tested according to the Italian Ministry of Health testing policies applied during the first months of the pandemic [14]. Those included close household and non-household contacts of symptomatic index cases under surveillance, healthcare professionals, and solicit testing in case of risk exposure. An asymptomatic case was defined as a person infected with SARS-CoV-2 who did not develop clinical symptoms and chest imaging findings of the disease (never symptomatic individual) [9]. The asymptomatic proportion was defined as the proportion of infected individuals who were never symptomatic for COVID-19 among the total number of infected individuals [15]. The asymptomatic SARS-CoV-2 RNA positive period was defined as the number of days between the first positive PCR test and the first of the two serial negative PCR tests.

Categorical variables were summarized as the counts and percentages in each category. Continuous variables were expressed as the medians and interquartile ranges (IQR). KruskalWallis test was applied to continuous variables. Binary and multivariate logistic regression analyses were performed to evaluate whether cases' demographics and clinical characteristics were independently associated with having asymptomatic infection. Analysis was conducted with STATA/SE 15.0 .

As this study constituted public health surveillance, ethical approval from institutional review board was not required. All data were provided and analyzed anonymously.

\section{Results}

Between February 29 and July 7, 2020, a total of 4,536 cases (51.1\% male; median age: 56 years, IQR: 41-72; cumulative incidence: 115.5 per 100,000 inhabitants) were diagnosed with COVID-19 in the Apulia region, Italy.

The group of persons with asymptomatic SARS-CoV-2 infection consisted of $903(53.5 \%$ female) cases among 193,757 tests performed, with median age of 50 years (IQR: 32-63). Of 335 cases for whom this information was only available, $146(43.6 \%)$ had underlying medical conditions, the most prevalent being cardiovascular disease $(24.8 \%)$, neurological diseases $(6.6 \%)$, diabetes $(4.2 \%)$ and chronic lung disease $(3 \%)$. Comparison of demographics and clinical characteristics of asymptomatic versus symptomatic presentation following SARSCoV-2 infection are shown in Table 1. 
Table 1: Comparison of characteristics between asymptomatic and symptomatic SARS-CoV-2 infections, Apulia region, Italy, February-July 2020.

\begin{tabular}{|c|c|c|c|c|c|c|c|c|c|c|c|c|}
\hline \multirow{3}{*}{$\begin{array}{l}\text { Characteristics } \\
\text { Sex }\end{array}$} & \multirow{2}{*}{\multicolumn{2}{|c|}{$\begin{array}{l}\text { Asymptomatic SARS- } \\
\text { CoV-2 infections } \\
\text { (no. 903) }\end{array}$}} & \multirow{2}{*}{\multicolumn{2}{|c|}{$\begin{array}{c}\text { Symptomatic SARS-CoV- } \\
2 \text { infections } \\
\text { (no. } 3,633 \text { ) }\end{array}$}} & \multirow{2}{*}{\multicolumn{2}{|c|}{$\begin{array}{c}\text { Total } \\
\text { (no. } 4,536)\end{array}$}} & \multicolumn{6}{|c|}{ Logistic regression analysis } \\
\hline & & & & & & & \multicolumn{3}{|c|}{ Binary } & \multicolumn{3}{|c|}{ Multivariate } \\
\hline & no. & $\%$ & no. & $\%$ & no. & $\%$ & OR & $95 \% \mathrm{CI}$ & $\mathrm{p}$ & OR & $95 \% \mathrm{CI}$ & $\mathrm{p}$ \\
\hline Female & 483 & 53.49 & 1,734 & 47.73 & 2,217 & 48.88 & Ref. & & & Ref. & & \\
\hline Male & 420 & 46.51 & 1,899 & 52.27 & 2,319 & 51.12 & 0.79 & $0.69-0.92$ & 0.002 & 0.69 & $0.54-0.86$ & 0.002 \\
\hline \multicolumn{13}{|l|}{ Age group, years } \\
\hline $0-9$ & 28 & 3.10 & 47 & 1.29 & 75 & 1.65 & Ref. & & & Ref. & & \\
\hline $10-19$ & 53 & 5.87 & 80 & 2.20 & 133 & 2.93 & 1.11 & $0.62-1.99$ & 0.721 & & & \\
\hline $20-29$ & 98 & 10.85 & 284 & 7.82 & 382 & 8.42 & 0.58 & $0.34-0.97$ & 0.040 & & & \\
\hline $30-39$ & 125 & 13.84 & 370 & 10.18 & 495 & 10.91 & 0.56 & $0.34-0.94$ & 0.029 & & & \\
\hline $40-49$ & 137 & 15.17 & 499 & 13.74 & 636 & 14.02 & 0.46 & $0.28-0.76$ & 0.003 & & & \\
\hline $50-59$ & 189 & 20.93 & 696 & 19.16 & 885 & 19.51 & 0.45 & $0.28-0.75$ & 0.002 & 0.89 & $0.83-0.96$ & 0.001 \\
\hline $60-69$ & 100 & 11.07 & 560 & 15.41 & 660 & 14.55 & 0.3 & $0.18-0.50$ & $<0.001$ & & & \\
\hline $70-79$ & 63 & 6.98 & 436 & 12.00 & 499 & 11.00 & 0.24 & $0.14-0.41$ & $<0.001$ & & & \\
\hline $80-89$ & 77 & 8.53 & 467 & 12.85 & 544 & 11.99 & 0.28 & $0.16-0.46$ & $<0.001$ & & & \\
\hline$\geq 90$ & 33 & 3.65 & 194 & 5.34 & 227 & 5.00 & 0.27 & $0.16-0.52$ & $<0.001$ & & & \\
\hline \multicolumn{13}{|l|}{ Single Comorbidities* } \\
\hline None & 189 & 56.42 & 676 & 35.39 & 865 & 38.53 & Ref. & & & Ref. & & \\
\hline Cardiovascular disease & 83 & 24.78 & 733 & 38.38 & 816 & 36.35 & 0.41 & $0.37-0.54$ & $<0.001$ & & & \\
\hline Diabetes & 14 & 4.18 & 244 & 12.77 & 258 & 11.49 & 0.21 & $0.12-0.36$ & $<0.001$ & & & \\
\hline Chronic pulmonary disease & 11 & 3.28 & 232 & 12.15 & 243 & 10.82 & 0.17 & $0.09-0.32$ & $<0.001$ & & & \\
\hline Cancer & 9 & 2.69 & 141 & 7.38 & 150 & 6.68 & 0.23 & $0.11-0.46$ & $<0.001$ & & & \\
\hline Neurological diseases & 22 & 6.57 & 178 & 9.32 & 200 & 8.91 & 0.44 & $0.28-0.71$ & 0.001 & & & \\
\hline Chronic kidney disease & 3 & 0.90 & 110 & 5.76 & 113 & 5.03 & 0.1 & $0.03-0.31$ & $<0.001$ & & & \\
\hline Obesity & 5 & 1.49 & 89 & 4.66 & 94 & 4.19 & 0.2 & $0.08-0.5$ & 0.001 & & & \\
\hline Other metabolic diseases & 10 & 2.99 & 58 & 3.04 & 68 & 3.03 & 0.62 & $0.31-1.22$ & 0.170 & & & \\
\hline Liver disease & 2 & 0.60 & 33 & 1.73 & 35 & 1.56 & 0.22 & $0.05-0.91$ & 0.037 & & & \\
\hline HIV & 2 & 0.60 & 26 & 1.36 & 28 & 1.25 & 0.27 & $0.06-1.17$ & 0.081 & & & \\
\hline At least one comorbidity & 146 & 43.58 & 1,234 & 64.61 & 1,380 & 61.427 & 0.42 & $0.33-0.53$ & $<0.001$ & 0.55 & $0.41-0.73$ & $<0.001$ \\
\hline
\end{tabular}

Ref., reference group.

* 2,245 cases (335 asymptomatic and 1,910 symptomatic infections) for whom the information was available. 
The asymptomatic proportion was estimated to be $19.9 \%$ (95\% confidence interval (CI): 18.8-21.1\%). The median asymptomatic SARS-CoV-2 RNA positive period was 19 days (IQR: 14-31) and the cumulative proportion of persons with resolution of infection 14 days after the first positive PCR test was 74\% (Figure 1).

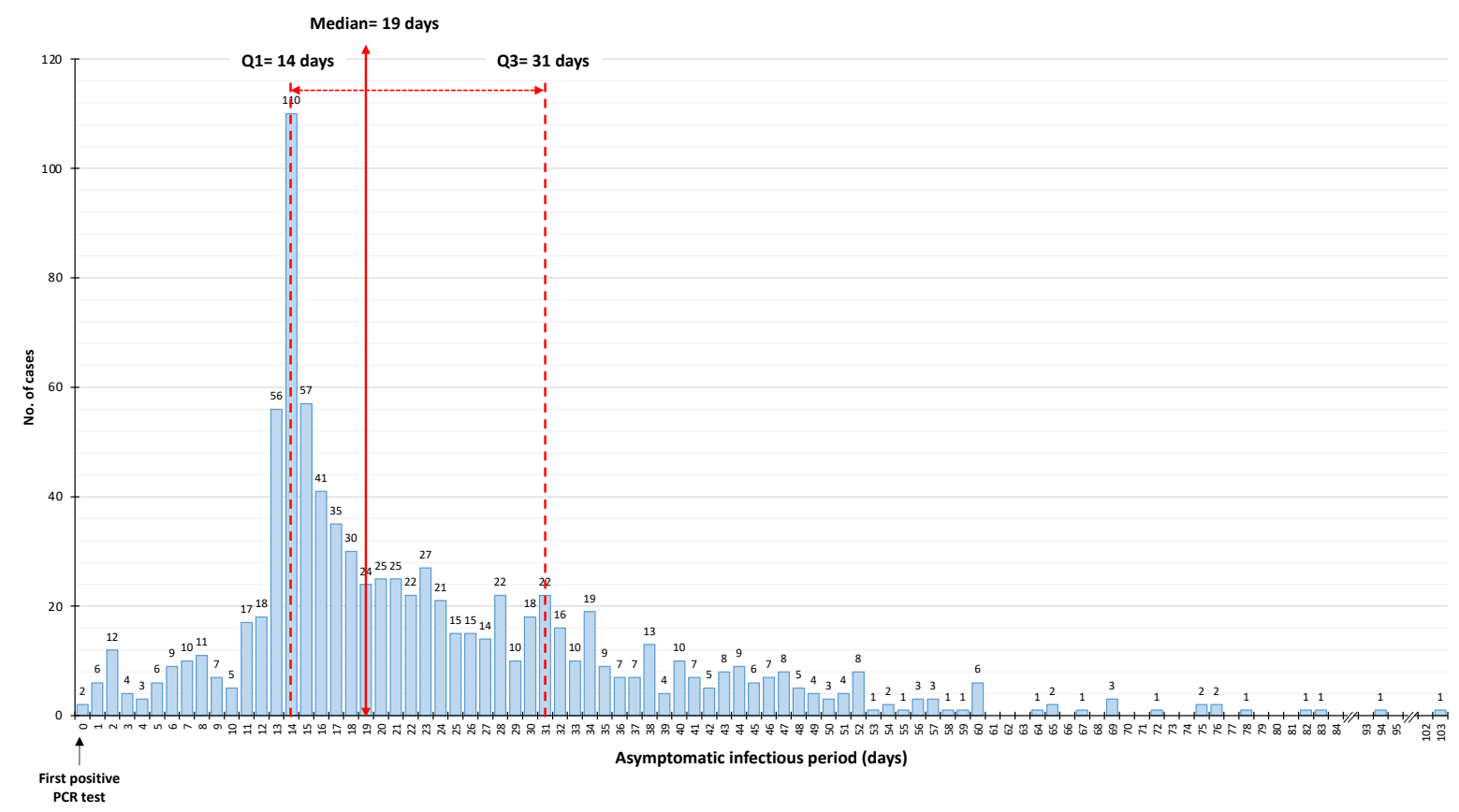

Figure 1: Asymptomatic SARS-CoV-2 RNA positive period, Apulia region, Italy, February-July 2020.

The probability of having asymptomatic infection decreased with increasing age (Odd Ratio for being asymptomatic with each 10-year increase in age: $0.89,95 \%$ CI: $0.83-0.96$, $\mathrm{p}=0.001$ ), in individuals with underlying diseases (OR: 0.55, 95\% CI:0.41-0.73, $\mathrm{p}<0.001$ ), and in males (OR: 0.69, 95\% CI: 0.54-0.86, $\mathrm{p}=0.002$ ) (Table 1).

The risk of delayed resolution of infection increased with increasing age, with median asymptomatic SARS-CoV-2 RNA positive period increasing from 16.5 days (IQR:14-26) in persons aged 0-9 years to 28 days (IQR: $18-40)$ in those aged $80-89$ years $(\mathrm{p}<0.001)$.

\section{Discussion}

After the end of the first epidemic wave of COVID-19 outbreak in Italy, we tried to estimate the proportion of people with SARS-CoV-2 who were asymptomatic. Findings from our study in the Apulia region showed that between $18.8 \%$ and $21.1 \%$ of people testing positive for SARS-CoV-2 during the appropriate follow-up actions were asymptomatic. These estimates were consistent with the pooled percentages of asymptomatic infections estimated in several systematic reviews and meta-analyses performed so far during the pandemic (Table 2). 
Table 2. The proportion of asymptomatic SARS-CoV-2 infections reported in different systematic reviews and meta-analyses.

\begin{tabular}{|c|c|c|c|c|}
\hline First author & Study period & $\begin{array}{r}\text { Total SARS-CoV-2, } \\
\text { no. }\end{array}$ & $\begin{array}{r}\text { Asymptomatic SARS-CoV-2, } \\
\text { no. }\end{array}$ & Pooled proportions \\
\hline A. Kronbichlera, et al. [16] & $\begin{array}{l}\text { December 1, } 2019 \text { to } \\
\text { March 29, } 2020\end{array}$ & NR & $\begin{array}{ll}506 \\
\end{array}$ & $\begin{array}{r}24.2 \% \\
(\mathrm{SD} 22.06) \\
\end{array}$ \\
\hline J. Zhu, et al. [17] & $\begin{array}{l}\text { January } 1 \text { to February } \\
28,2020\end{array}$ & 3,062 & 158 & $\begin{array}{r}11.9 \% \\
(95 \% \text { CI: } 2.9-25.8 \%) \\
\end{array}$ \\
\hline C. Chen, et al. [18] & $\begin{array}{l}\text { January } 1 \text { to May } 13, \\
2020\end{array}$ & 20,152 & NR & $\begin{array}{r}13.34 \% \\
(95 \% \text { CI: } 10.86-16.29 \%) \\
\end{array}$ \\
\hline J. He, et al. [8] & Before May 20, 2020 & 50,155 & 1,430 & $\begin{array}{r}15.6 \% \\
\text { (95\% CI: } 10.1-23.0 \%) \\
\end{array}$ \\
\hline $\begin{array}{l}\text { D. Buitrago-Garcia, et al. } \\
\text { [19] }\end{array}$ & $\begin{array}{l}\text { March } 25 \text { to June 10, } \\
2020\end{array}$ & 6,616 & 1,287 & $\begin{array}{r}20 \% \\
(95 \% \text { CI: } 17-25 \%) \\
\end{array}$ \\
\hline M. Alene, et al. [20] & $\begin{array}{l}\text { June } 1 \text { to December 9, } \\
2020\end{array}$ & 6,071 & 1,917 & $\begin{array}{r}25 \% \\
\text { (95\% CI: } 16-38 \%) \\
\end{array}$ \\
\hline P. Sah, et al. [21] & $\begin{array}{l}\text { January } 1,2020 \text { to April } \\
2,2021\end{array}$ & 17,272 & 7,222 & $\begin{array}{r}35.1 \% \\
(95 \% \text { CI: } 30.7-39.9 \%)\end{array}$ \\
\hline
\end{tabular}

NR, not reported

$\mathrm{SD}$, standard deviation 
However, the Lavezzo et al. prospective cohort study conducted in the Italian municipality of Vò reported much higher proportion of asymptomatic infections (42.5\%) [22]. This difference could be explained by the fact that in our study we analyzed a larger number of cases. Moreover, the retrospective nature of our study allowed us to exclude from the analysis the asymptomatic individuals who developed symptoms later during follow-up. In fact, presymptomatic patients are easily misclassified as asymptomatic if the follow-up is not long enough and this may lead to overestimate the true burden of asymptomatic infection [8].

From the beginning of the pandemic of SARS-CoV-2, older age, prior illnesses and male sex have emerged as risk factors [8]. Our results showed that asymptomatic infections were more common in young individuals without underlying diseases and among females. A metaanalysis for studies with younger COVID-19 positive populations (e.g., obstetric patients) found that in these younger populations it appeared fewer people developed symptoms compared to older groups, during similar follow up times [23]. In Italy, this could be attributed to an underestimation of younger COVID-19 cases in the early phase of the outbreak due to various detection policies that led to the restriction of testing with swab to mainly symptomatic cases. Following the period examined in this study, when the World Health Organization guidelines were relaxed and Italy entered the transition phase, the testing policy has been broadened to various screening programmes (e.g., ahead of hospital admission for other causes, diagnostic suspicion that emerge during clinical activities, screening/tracing activities that emerge via planned tests of travellers returning to Italy from foreign countries with higher virus circulation, immigrants, and employees) [4]. As a result, between July and mid-August 2020, when the second wave pandemic started, it was reported that overall $76 \%$ of asymptomatic cases seen in the Apulia region were aged between 0 and 50 years (median age: 28 years). It is likely that also the difference between the medians of age of asymptomatic cases in the two consecutive periods ( $50 \mathrm{vs.} 28$ years) reflected the sensitivity of case finding and diagnostic protocols rather than the true disease epidemiology.

Our data showed that one in four asymptomatically infected person had a positive nucleic test result to more than four weeks after the first test (Figure 1), this may indicate that the longterm infection of SARS-CoV-2 might really do exist among asymptomatic cases. In particular, long-term carrying of the virus was more common for asymptomatic older cases than the youngest cases.

This study had two main limitations. First, in view of the reasons mentioned above, asymptomatic cases in our first outbreak cohort could have represented a peculiar, underestimated fraction of the SARS-CoV-2 asymptomatic transmission. Since the beginning of the mitigation stage in late February 2020, most Italian regions experienced a severe lack of testing material and capacity leading the Italian Ministry of Health to prioritize viral RNA testing for symptomatic people and subjects at high-risk for Sars-CoV-2 infections while drastically reducing testing of the asymptomatic general population [24, 25].

Second, the peak of resolution of infection observed at 14 days (Figure 1) was an artefact of the surveillance and depended on the fact that many cases were tested at day 14 before exiting the quarantine period. Anyhow, such peak did not affect either median or Q3 value calculation that were very likely to be 19 and 31 days respectively. 


\section{Conclusions}

Identifying asymptomatic cases has been of crucial importance for SARS-Cov-2 in the subsequent outbreak phases. In Italy, the national weekly epidemiological bulletins reported that asymptomatic infections have represented an increasing fraction of the infected population over time ( $>50 \%$ [26]), also as a result of increased testing capacity for COVID19. The presence of a large proportion of asymptomatic and late spreaders of the virus has continued to be a challenge for controlling the pandemic. Atripaldi et al. suggested that, in Italy, the rapid spread of the second outbreak since late August could have been linked to the circulation of asymptomatic individuals and to their consistent contribution to community transmission in post lockdown [27]. They might also be contributing substantially to building herd immunity in addition to the real-life effect of the ongoing mass vaccination campaign (including indirect protection). However, further research is required to learn more about on how the virus adapts to humans and its future management, including COVID-19 surveillance, strategic testing policies and public health interventions. 


\section{Data Availability}

The data that support the findings of this study are available from the Apulia Public Health Authority, but restrictions apply to the availability of these data, which were used under license for the current study, and so are not publicly available. Data are however available from the authors upon reasonable request and with permission of the Apulia Public Health Authority.

\section{Conflicts of Interest}

The authors declare that there is no conflict of interest regarding the publication of this paper.

\section{Funding Statement}

No sponsorship or funding was received for this study or for the publication of this article.

\section{Acknowledgments}

The Authors would like to thank all the patients whose data were used in this study. A special thanks to all the frontline health workers: staff at Regional Public Health Authority, staff at Local Health Units, staff at Hospitals, and staff at Laboratories across the Apulia region, for their dedication and valuable work into pandemic control.

An earlier version of this manuscript has been presented as a preprint in the following link: https://www.researchsquare.com/article/rs-93013/v1 [28]. Portion of this study data were presented as oral communication at the XLV Conference of the Italian Association of Epidemiology, on-line edition, held on 26-30 April 2021. 


\section{References}

[1] J. Chen. "Pathogenicity and transmissibility of 2019-nCoV-A quick overview and comparison with other emerging viruses," Microbes and Infection, vol. 22, no. 2, pp. 69-71, 2020.

[2] World Health Organization, "Coronavirus disease (COVID-19) Situation Report - 169." 2020 Jul 7, https://www.who.int/docs/default-source/coronaviruse/situation-reports/20200707-covid-19-sitrep169.pdf? sfvrsn=c6c69c88.

[3] EpiCentro - Epidemiology for Public Health. Italian National Institute of Health (Istituto Superiore di Sanità), "COVID-19 integrated surveillance data in Italy, 2020 Jun 30",

https://www.epicentro.iss.it/coronavirus/bollettino/Bollettino-sorveglianza-integrata-COVID-19 30giugno-2020.pdf.

[4] F. Riccardo, M. Ajelli, X.D. Andrianou, et al., "Epidemiological characteristics of COVID-19 cases and estimates of the reproductive numbers 1 month into the epidemic, Italy, 28 January to 31 March 2020,” Euro Surveillance, vol. 25, no. 49, Article ID 2000790, 2020.

[5] National Health Commission of People's Republic of China. "Diagnostic and treatment plan of Coronavirus disease 2019. 2020http://www.nhc.gov.cn/yzygj/s7653p/202003/46c9294

a7dfe4cef80dc7f5912eb1989.shtml tentative.

[6] World Health Organization. "Clinical management of severe acute respiratory infection (SARI) when COVID-19 disease is suspected", 2020, https://www.who.int/publications-detail/clinical-managementof-severe-acute-respiratory- infection-when-novel-coronavirus-(ncov)-infection-is-suspected

[7] M. Gandhi, D.S. Yokoem, D.V. Havlir, "Asymptomatic Transmission, the Achilles' Heel of Current Strategies to Control Covid-19," New England Journal of Medicine, vol. 382, no. 22, pp. 2158-2160, 2020.

[8] J. He, Y. Guo, R. Mao, J. Zhang, "Proportion of asymptomatic coronavirus disease 2019 (COVID-19): a systematic review and meta-analysis," Journal of Medical Virology, vol. 93, no. 2, pp. 820-830, 2021.

[9] Z. Gao, Y. Xu, C. Sun, X. Wang, Y. Guo, S. Qiu, K. Ma. "A systematic review of asymptomatic infections with COVID-19," Journal of Microbiology, Immunology and Infection, vo. 54, no 1, pp.12$16,2021$.

[10] S.W. Park, D.M. Cornforth, J. Dushoff, J.S. Weitz, "The time scale of asymptomatic transmission affects estimates of epidemic potential in the COVID-19 outbreak," Epidemics, vol. 31, Article ID $100392,2020$.

[11] A. Sakurai, T. Sasaki, S. Kato, et al., "Natural History of Asymptomatic SARS-CoV-2 Infection," New England Journal of Medicine, vol. 383, no. 9, pp. 885-886, 2020.

[12] World Health Organization, "Go.Data: Managing complex data in outbreaks", 2021, https://www.who.int/godata.

[13] P. Stefanelli, G. Faggioni, A. Lo Presti, et al., "Whole genome and phylogenetic analysis of two SARSCoV-2 strains isolated in Italy in January and February 2020: additional clues on multiple introductions and further circulation in Europe," Euro Surveillance, vol. 25, no. 13, Article ID 2000305, 2020.

[14] G. Onder, G.Rezza, S. Brusaferro. "Case-Fatality Rate and Characteristics of Patients Dying in Relation to COVID-19 in Italy," JAMA, vol. 323, no. 18, pp. 1775-1776, 2020. 
[15] K. Mizumoto, K. Kagaya, A. Zarebsky, G Chowell, "Estimating the asymptomatic proportion of coronavirus disease 2019 (COVID-19) cases on board the Diamond Princess cruise ship, Yokohama, Japan, 2020,”Euro Surveillance, vol. 25, no. 10, Article ID 2000180, 2020.

[16] A. Kronbichlera, D. Kresse, S. Yoonc, K.H. Leed, M. Effenbergere, J.I. Shind. "Asymptomatic patients as a source of COVID-19 infections: A systematic review and meta-analysis," International Journal of Infectious Diseases, vol. 98, pp. 180-186.

[17] J. Zhu, P, Ji, J. Pang, Z. Zhong, H. Li, C. He, J. Zhang, C. Zhao. "Clinical characteristics of 3062 COVID-19 patients: A meta-analysis,” Journal of Medical Virology, vol. 92, no. 10, pp. 1902-1914.

[18] C. Chen, C. Zhub, D. Yana, H. Liub, D. Lib, Y. Zhoua, X. Fua, et a. "The epidemiological and radiographical characteristics of asymptomatic infections with the novel coronavirus (COVID-19): A systematic review and meta-analysis," International Journal of Infectious Diseases, vol. 104, pp. 458464.

[19] D. Buitrago-Garcia, D. Egli-Gany, M.J. Counotte, S. Hossmann, H. Imeri, 1, A.M. Ipekci, et al. "Occurrence and transmission potential of asymptomatic and presymptomatic SARSCoV-2 infections: A living systematic review and meta-analysis," PLoS Medicine, vol. 17, no. 9, pp. e1003346.

[20] M. Alene, L. Yismaw, M.A. Assemie, D.B. Ketema, B. Mengist, et al. "Magnitude of asymptomatic COVID-19 cases throughout the course of infection: A systematic review and meta-analysis," PLoS One, vol. 16, no. 3, pp. e0249090.

[21] P. Sah, M.C. Fitzpatrick, C.F. Zimmera, E.Abdollahic, L. Juden-Kellyc, S.M. Moghadasc, et al. "Asymptomatic SARS-CoV-2 infection: A systematic review and meta-analysis," Proceedings of the National Academy of Sciences, vol. 118, no. 34, pp. e2109229118

[22] E. Lavezzo, E. Franchin, C. Ciavarella, et al., "Suppression of a SARS-CoV-2 outbreak in the Italian municipality of Vo'," Nature, vol. 584, no. 7821, pp. 425-429, 2020.

[23] M. Yanes-Lane, N. Winters, F. Fregonese, et al., "Proportion of asymptomatic infection among COVID-19 positive persons and their transmission potential: A systematic review and meta-analysis." PLoS One, vol. 15, no. 11, Article ID e0241536, 2020.

[24] A. De Matteis, F.B. Turkmen Ceylan, M. Adrianopoli, S.Ertek. "The contribution of testing in the fight against COVID-19: evidence from Italy.” The European Journal of Public Health, vol. 31, no. 4, pp. 913-917, 2021.

[25] M. Di Bari, D. Balzi, G. Carreras, G. Onder. "Extensive Testing May Reduce COVID-19 Mortality: A Lesson From Northern Italy," Frontiers in Medicine, vol. 7, pp. 402, 2020

[26] EpiCentro - Epidemiology for Public Health. Italian National Institute of Health (Istituto Superiore di Sanità), "COVID-19 integrated surveillance data in Italy," 2021 Jun 30,

https://www.epicentro.iss.it/coronavirus/bollettino/Bollettino-sorveglianza-integrata-COVID-19_30giugno-2021.pdf

[27] L. Atripaldi, S. Sale, M. Capone, et al., "Could asymptomatic carriers spread the SARS-CoV-2 infection? Experience from the Italian second wave," Journal of Translational Medicine volume, vol. 1, no. 1, pp 93, 2021.

[28] D. Martinelli, F. Fortunato, S. Mazzilli, et al., "Estimating the proportion of asymptomatic COVID-19 cases during the first pandemic wave in Apulia region, Italy", 2020,

https://www.researchsquare.com/article/rs-93013/v1 
Figures

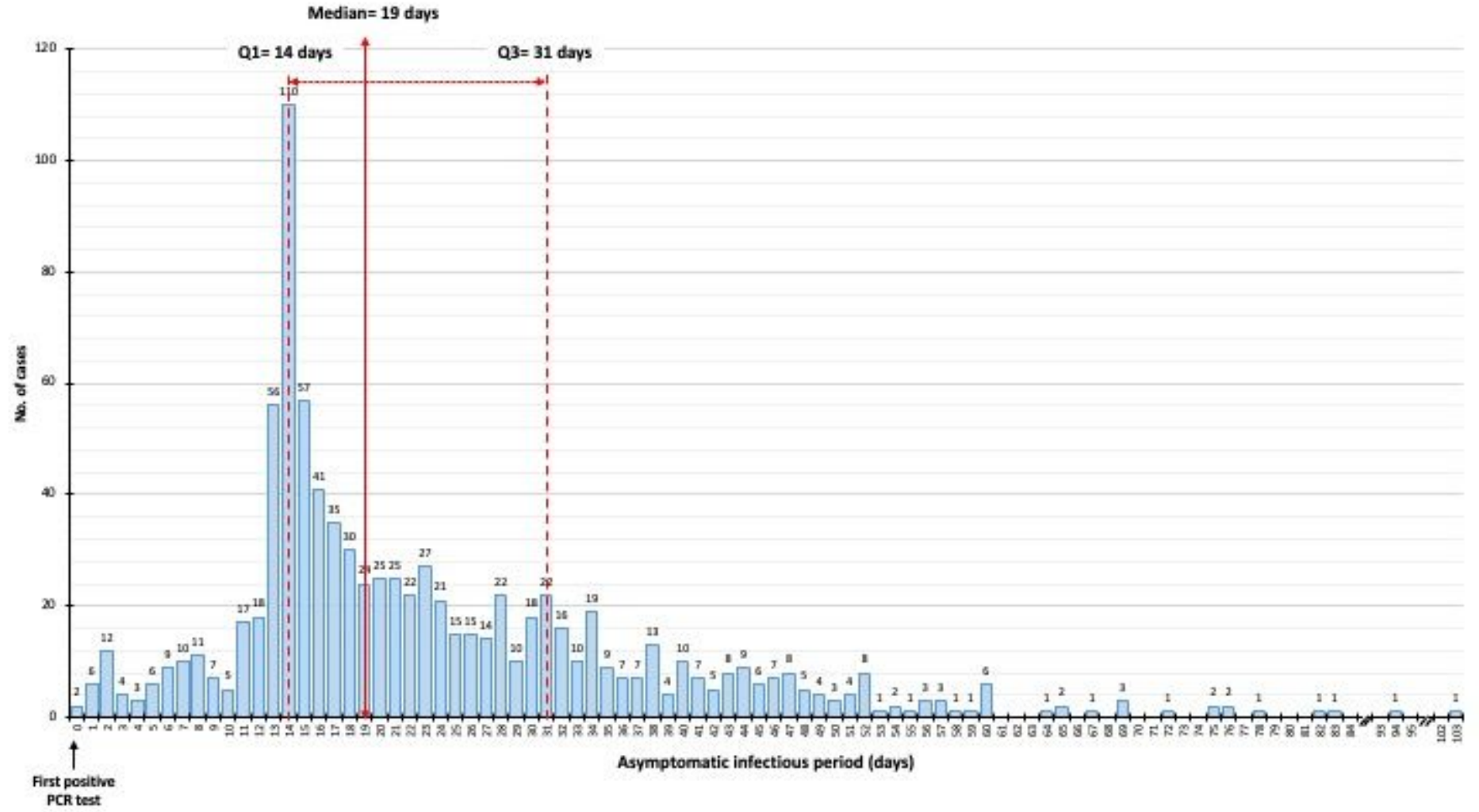

Figure 1

Asymptomatic SARS-CoV-2 RNA positive period, Apulia region, Italy, February-July 2020. 